www.jmscr.igmpublication.org

Impact Factor (SJIF): 6.379

Index Copernicus Value: 79.54

ISSN (e)-2347-176x ISSN (p) 2455-0450

crossrefDOI: https://dx.doi.org/10.18535/jmscr/v6i11.82

Journal Of Medical Science And Clinical Research

\title{
Evaluation of Effects of Hypo/Hyperthyroidism on Glycated Haemoglobin in Euglycaemic Patients
}

\author{
Authors \\ Dr Umesh Chandra Jha ${ }^{1}$, Dr Peyalee Sarkar ${ }^{2}$ \\ ${ }^{1}$ Associate professor, Dept of Medicine, DMCH, Laheriasarai \\ ${ }^{2} 3^{\text {rd }}$ year post graduate trainee, Dept of Medicine, DMCH, Laheriasarai
}

\begin{abstract}
American Diabetes Association has suggested the use of HbAlc as diagnostic tool for prediabetes and diabetes. A value between $5.7 \%$ and $6.5 \%$ represents prediabetes while a value $\geq 6.5 \%$ is considered as diabetes mellitus. However, several factors other than glycemic status can influence HbAlc levels, factors which affect the RBC turnover like Haemoglobinopathies (sickle cell anemia etc.), even Blood transfusion and as Thyroid status affects cell turnover, it is being hypothesised that thyroid status changes( hypo or hyperthyroid) will cause raised levels of HB1AC levels. Raised HBIAC levels in thyroid disorders can falsely classify patients as prediabetic. The aim of the study was to determine the effects of altered thyroid status on HbAlc levels in individuals without diabetes, with overt hyper- and hypo-thyroidism. All newly diagnosed cases matching the inclusion criteria(attending the outdoors of medicine department, DMCH, Laheriasarai) with thyroid disorders between 1st april 2017 till 31st october 2018 in Department of Medicine, DMCH were included in the study, with equal numbers of age and sex matched controls. Results showed an increased level of HBAIC in patients with thyroid disorders and the presumed conclusion was that in patients with thyroid disorders glycated haemoglobin is not an assured marker of imapired glucose metabolism and other methods should be used.
\end{abstract}

\section{Introduction}

Diabetes is one of the largest global health epidemics of the 21 st century. During the past twenty years the number of people with diabetes worldwide has more than doubled. One of the worst worrying features of this rapid increase in the emergence of type $2 \mathrm{DM}$ in children, adolescents and young adults, whereas the disease was once confined mainly to older adults. Diabetes mellitus is a leading cause of death and disability worldwide. It's global prevalence was about $8.8 \%$ in 2017 and is predicted to rise to 9.9\% in 2045. a major contributor to the challenge of preventing the complications associated with diabetes is that a high proportion $20 \%-50 \%$ of people with diabetes are undiagnosed. China is likely home to the largest number of adults with diabetes (114.4 million) followed by India (72.9 million). The International Diabetes Federation diabetes atlas provides predictions for the top 10 countries in the world with the highest number of people with diabetes in 2017 and expected numbers in 2045. The most recent is that India will be the country with the highest number of people expected to have diabetes. 
Criteria for diagnosis of diabetes

1) Symptoms of diabetes plus random blood glucose concentration $\geq 11.1 \mathrm{mmol} / \mathrm{L}(200$ $\mathrm{mg} / \mathrm{dL})$ or

2) Fasting plasma glucose $\geq 7.0 \mathrm{mmol} / \mathrm{L}$ (126 $\mathrm{mg} / \mathrm{dL}$ ) or

3) Haemoglobin A1c $\geq 6.5 \% \mathrm{c}$ or

4) $2 \mathrm{~h}$ plasma glucose $\geq 11.1 \mathrm{mmol} / \mathrm{L}$ (200 $\mathrm{mg} / \mathrm{dL}$ ) during an oral glucose tolerance test with $75 \mathrm{~g}$ OGTT

Glycated hemoglobin (A1c): HbA1C

Haemoglobin is the oxygen-carrying pigment that gives blood its red colour and is also the predominant protein in red blood cells. About $90 \%$ of haemoglobin is haemoglobin A (the "A" stands for adult type). Although one chemical component accounts for $92 \%$ of haemoglobin A, approximately $8 \%$ of haemoglobin A is made up of minor components that are chemically slightly different. These minor components include haemoglobin A1c, A1b, A1a1, and A1a2. Haemoglobin A1c (HbA1c) is a minor component of haemoglobin to which glucose is bound. HbAlc also is sometimes referred to as glycated, glycosylated haemoglobin, or glycohaemoglobin.

Normal value is $<5.4 \%$.... When more than $6.5 \%$ on two separate occasions it is diagnostic of Diabetes and is the most accurate test for the above.

The major form of the glycated haemoglobin is haemoglobin A1c (HbA1c). The HbA1c concentration not only depends on prevailing glycaemia but also the life span of the erythrocytes and so, the conditions which affect the erythrocyte turnover or survival lead to falsely high or low HbA1c levels. A study conducted by Kim MK et al., showed that even in the absence of diabetes, there was spurious elevation of $\mathrm{HbA1c}$ in patients with hypothyroidism. Thyrotoxicosis is known to cause increased RBC turnover. Hypothyroidism has opposite effect. We therefore hypothesize that A1c level does not accurately reflect glycemia in hypothyroidism and thyrotoxicosis. Both of these thyroid disorders are widely prevalent worldwide. For this reason it is very important to know the influence of altered thyroid status on Glycated hemoglobin.

\section{Aims and Objectives}

1. To evaluate the effect of hypo and hyperthyroidism on glycated haemoglobin (HBA1C) in euglycaemic non-diabetic patients.

2. Assess the effectiveness of glycated haemoglobin as a diagnostic tool for diabetes in patients with thyroid disorders.

\section{Methods and Materials}

A cross sectional observational study was conducted amongst the patients attending the general medicine outdoor of Darbhanga Medical College and Hospital, Darbhanga for a study period of 1.5 years $\left(1^{\text {st }}\right.$ April $2017-31^{\text {st }}$ October 2018).

\section{Inclusion Criteria}

- Age between 18 to 60 years of both the genders

- Recently diagnosed overt primary hypothyroidism or hyperthyroidism $(<3$ months)

\section{Exclusion Criteria}

- Patients with diabetes, IGT, or IFG

- Hemoglobin <10 gm/dl

- Renal failure (Creatinine clearance< $60 \mathrm{ml} / \mathrm{min}$ )

- Hepatic dysfunction (increased bilirubin, reduced albumin $[<3.5 \mathrm{mg} / \mathrm{dl}]$, SGOT and SGPT 3 times upper limit of normal)

- Acute or subacute thyroiditis For statistical analysis SPSS version 21 was used.

\section{Review of Literature}

\section{Glycated Hemoglobin}

Normal adult hemoglobin ( $\mathrm{HbA})$ is made up of four chains of amino acids $(2 \alpha, 2 \beta)$. Glycation of hemoglobin can occur on the alpha or beta chain, and at different points in the chains. This results in 
a 'family' of glycated hemoglobins. Total glycated hemoglobin includes all hemoglobin that has reacted with a sugar. They are collectively known as $\mathrm{HbA} 1$ and classified further according to their order of elution as HbAla, HbA1b and $\mathrm{HbA1c}$. Last one is the major fraction of $\mathrm{HbA} 1$. It is a hemoglobin $(\mathrm{Hb})$ molecules with a stable adduct of glucose to the $\mathrm{N}$-terminal valine of the $\mathrm{Hb} \beta$ chain $[\beta-\mathrm{N}(1$-deoxy)fructosyl-Hb]. The term 'glycosylated' was used initially, but it has been pointed out that this term strictly refers to glycosides. Therefore, the Joint Commission on Biochemical Nomenclature has proposed that the term 'glycation' is appropriate for any reaction that links a sugar to a protein. In the particular case of a reaction with hemoglobin, the term 'glycated hemoglobin' is justified. In current literatures, the terms 'glycated hemoglobin', HbA1c, and A1c are used interchangeably.

\section{History}

Stable ketoamine adduct formation following reaction of reducing sugars with amino acids was described by L C Maillard in 1912. In 1958, Allen et al demonstrated that normal adult hemoglobin could be separated chromatographically on a cation exchange resin into a major component, $\mathrm{A}_{0}$, accounting for more than $90 \%$ of the hemoglobin and three negatively charged minor components, which they designated as HbA1a, HbAlb and HbA1c, (collectively known as HbA1). ${ }^{6}$ In 1960s, Rahbar et al identified A1c as an "unusual hemoglobin in patients with diabetes".Around the same time, there was a strong suspicion that hyperglycemia was related to the vascular complications observed in individuals with diabetes, but the association was difficult to prove due to a lack of objective markers of glucose control.Trivelli et al found a two-fold increase of A1c over values observed in non-diabetic subjects. ${ }^{9}$ It was introduced into wide-spread clinical use in the late1970s and subsequently became a cornerstone of clinical practice. The Diabetes Control and Complications Trial (DCCT) and UK Prospective Diabetes Study
(UKPDS) demonstrated that in Type 1 and Type 2 diabetes, respectively, intensive glucose control, reflected in blood glucose and A1c measurements, decreased the risk of complications. Furthermore, data on the correlation between blood glucose levels and A1c from these studies were used to derive regression equations for calculating average blood glucose (the mean of the seven daily capillary glucose recordings for the preceding 120 days, as described in the DCCT) from the A1c, aiding in the formation of current diabetes management guidelines.

A1c is affected by a number of genetic, hematologic, medicines, and illness-related factors. Changes in erythrocyte lifespan can affect A1c, because increasing the mean age of erythrocytes will increase A1c. Although certain disease states will alter the erythrocyte lifespan, there appears to be significant inter-individual variation in mean erythrocyte age in those without known hematological disorders, potentially accounting for some of the variation in A1c in individuals without diabetes. An increase in the mean age of erythrocytes will occur in the setting of decreased erythropoiesis, such as in iron and vitamin B12 deficiency, due to a lack of erythropoietin in renal failure, and due to bone marrow suppression in alcoholism. Alteration of tertiary structure of globin leading to increased glycation has been proposed as another mechanism of inappropriately elevated A1c level. Conversely, decrease the erythrocyte mean age will decrease A1c. This is seen with hemolytic anemia, after administration of erythropoietin in patients with renal failure, and after repletion of iron and vitamin B12 stores. Increased reticulocytes and a lower A1c are also seen in chronic liver disease, even in the absence of cirrhosis and splenomegaly, but the mechanism responsible is uncertain. Increased rates of hemolysis from splenomegaly, rheumatoid arthritis, or drugs such as anti-retrovirals, ribavirin, and dapsone can lead to decreased A1c. Splenectomy increases A1c as a result of increased erythrocyte survival. 
Kim MK et al., study suggested that the HbA1c levels decreased after thyroid hormone replacement in the patients with overt hypothyroidism while, the serum Erythropoietin (EPO) level, reticulocyte count and $\mathrm{MCH}$ increased after thyroid hormone replacement, suggesting that thyroid hormone stimulates erythropoiesis. These data also suggest that the thyroid hormone replacement is associated with a decrease in the HbA1c level, which is influenced by increased erythropoiesis rather than by the changes in glucose level. Christy AL et al., found that the non-diabetic hypothyroid individuals with anemia showed the elevated HbA1c levels in prediabetes range and concluded that the elevated HbA1c in hypothyroidism can be attributed to anaemia. Billic-Komarica $\mathrm{E}$ et al., also reported that the correlation between the level of serum TSH and HbA1c was positive and significant $(\mathrm{r}=0.46)$. Hypothyroidism independently increases the risk for decreased insulin sensitivity, especially in the adipose tissue and muscle. There is an apparent correlation between the $\mathrm{SH}$ and hyperinsulinemia and insulin resistance.

\section{Results}

Among patients attending general medicine OPD of $\mathrm{DMCH}$, Darbhanga 55 patients with hypothyroidism and 50 patients with hyperthyroidism (thyrotoxicosis) were randomly selected. After applying exclusion criteria, 45 patients with hypothyroidism, 34 patients with hyperthyroidism and 46 controls were considered for final analysis. Age and gender of the patients in all the three groups were well matched with controls. Fasting and 2hour OGTT values were not significantly different between patients in either group and controls. Hemoglobin levels were also similar.

\begin{tabular}{|l|c|c|}
\hline & $\begin{array}{c}\text { Hypothyroid } \\
\mathrm{n}=45\end{array}$ & $\begin{array}{c}\text { Control } \\
\mathrm{n}=46\end{array}$ \\
\hline Age(SD) & 44.3 & 40.6 \\
\hline Gender (M/F) & $13 / 32$ & $13 / 33$ \\
\hline FPG & 89.4 & 93.4 \\
\hline PPPG & 106.8 & 111.6 \\
\hline Hemoglobin & 12.1 & 12.4 \\
\hline
\end{tabular}

Fig. 1 Comparison of baseline characters between hypothyroid and control groups.
All values (except gender) have been elaborated as mean \pm standard deviation; $\mathrm{P}$ values were calculated by unpaired t-test; $\mathrm{P}<0.05$ was considered statistically significant

\begin{tabular}{|l|c|c|}
\hline & $\begin{array}{c}\text { Thyrotoxicosis } \\
\mathrm{n}=34\end{array}$ & $\begin{array}{c}\text { Control } \\
\mathrm{n}=46\end{array}$ \\
\hline Age(SD) & 38.3 & 40.6 \\
\hline Gender (M/F) & $9 / 25$ & $13 / 33$ \\
\hline FPG & 89.6 & 93.4 \\
\hline PPPG & 114.4 & 111.6 \\
\hline Hemoglobin & 12.4 & 12.4 \\
\hline
\end{tabular}

Fig. 2 Comparison of baseline characters between thyrotoxic and control groups.

As the A1c values were not normally distributed, Mann Whitney Rank sum test was used to compare mean A1c between the groups. When mean A1c values were compared between hypothyroid and control groups, it was found to be significantly higher in the former [Median +/inter-quartile range 5.6+/- 0.07 [hypothyroid] vs $5.2+/-0.04$ [controls]; $\mathrm{p}<.001$ ].

In contrast, mean A1c value did not differ significantly between thyrotoxic patients and controls [Median +/- inter-quartile range $5.3+/$ 0.5 [hyperthyroid] vs $5.2+/-0.04$ [controls]; $\mathrm{p}=$ $0.174]$.

\section{Conclusion}

1. Mean glycated hemoglobin level was found to be significantly higher in hypothyroid patients than control subjects despite similar glucose levels.

2. Significant difference in mean Glycated hemoglobin level was not observed between thyrotoxic patients and controls

3. Glycated haemoglobin may not be a reliable indicator of glycemic status in hypothyroid subjects and other markers of impaired glucose metabolism should be implemented for diagnosing diabetes mellitus.

\section{Reference}

1. Study of Glycated Haemoglobin (HbA1c) In Non-Diabetic Subjects with Subclinical Hypothyroidism Mayur Goradhanbhai 
Makadia, $\otimes^{1}$ Vishwal Indravadan Patel, journal of clinical and diagnostic research, 2017

2. The Importance of HbAlc Control in Patients with Subclinical Hypothyroidism Edina Billic-Komarica,, Materiasociomedica 2012.

3. Phillips P J et al. A1C - frequently asked questions. Australian Family Physician 2005: 34; 663-7.

4. Gallagher E J, Roith D L, Bloomgarden Z. Review of hemoglobin A1c in the management of diabetes.Journal of Diabetes 2009: 1; 9-17.

5. Jeppsson JO, Kobold U, ABarr $\mathrm{J}$ et al. Approved IFCC reference method for the measurement of HbA1c in human blood. Clin Chem Lab Med. 2002; 40: 78-89.

6. UK Prospective Diabetes Study (UKPDS) Group. Intensive blood-glucose control with sulphonylureas or insulin compared with conventional treatment and risk of complications in patients with type 2 diabetes (UKPDS 33). The Lancet. 1998

7. ADVANCE Collaboratrive Group . Intensive blood glucose and vascular outcomes in patients with type 2 diabetes . N Engl J Med 2008

8. Effects of thyroid hormone replacement on glycated hemoglobin levels in non diabetic subjects with overt hypothyroidism. Anantarapu $\mathrm{S}^{1}$, Vaikkakara S. arch endocrinol metab,2015

9. American Diabetes Association. Clinical practice recommendations 2012. Diabetes Care

10. Harrison's internal medicine $19^{\text {th }}$ edition

11. William's manual of endocrinology

12. National Collaborating Centre for Chronic Conditions. Type 2 Diabetes: National Clinical Guideline for Management in Primary and Secondary Care (Update) . London: Royal College of Physicians, 2008 .
13. International Expert Committee report on the role of the $\mathrm{A} 1 \mathrm{C}$ assay in the diagnosis of diabetes. Diabetes Care 2009.

14. Tahara Y, Shima K. Kinetics of HbA1c, glycated albumin, and fructosamine and analysis of their weight functions against preceding plasma glucose level. Diabetes Care 1995. 